unnecessary treatment. The aim of the study was to assess the impact of Specialist Palliative Care (SPC) review on reducing polypharmacy and prescribing anticipatory symptom control medication in inpatients at the end of life.

Methodology Adult inpatient deaths between July 2018 and February 2019 in an acute London Hospital were identified. Deaths in Intensive Care Unit and within 24 hours of admission were excluded. Demographic data, medication name and number on admission, at death and which were discontinued, were collected. Evidence of a SPC review and prescription for anticipatory medication were recorded. Using SPSS software, Chi Squared and Mann Whitney U tests were performed to identify statistical differences in prescribing for patients who had been reviewed by SPC.

Results 201 patients were identified. 159 patients (79\%) were reviewed by SPC, a median of 5 days prior to death (interquartile range $2-11$ days). Significantly more medications were discontinued in those who were reviewed by SPC (6.4 medications per patient) than those who were not reviewed (4.2 medications per patient, <0.001). 179 patients (89\%) were prescribed anticipatory medications. Of those reviewed by SPC, 158 (99\%) were prescribed these medications, therefore a SPC review made anticipatory medication prescription significantly more likely $(<0.001)$.

Conclusion SPC review reduced polypharmacy at the end of life and also increased the likelihood of anticipatory medication prescription. However, this mostly occurred only a few days prior to death. There needs to be continued focus on early identification of patients nearing end of life and medication review to enable appropriate deprescribing. Further research needs to determine whether deprescribing improves quality of life.

\section{WHAT ARE THE ATTITUDES OF PATIENTS RECEIVING PALLIATIVE CARE TOWARDS ASSISTED DYING?}

Kathryn L Jerram, Carol Davis. Cardiff University, Rowans Hospice

10.1136/spcare-2020-PCC.87

Research was undertaken for an MSc with the aim of gaining an idea of the attitudes of Hospice inpatients towards Assisted Dying (AD) and related subjects, in order to inform the current debate on legalising it in the UK. The study also aimed to identify whether there were associations between these attitudes and symptom and performance scores and whether or not these attitudes changed following inpatient Hospice care. This was a small pilot study using quantitative methods with a face to face questionnaire administered to Hospice inpatients soon after admission and two weeks later.

Small sample size limited statistical analysis, however it appears that a large majority of Hospice inpatients would agree with a change in the law to allow $\mathrm{AD}$ and many had considered measures to end their own lives in the past. Under half would have considered $\mathrm{AD}$ in the previous week, with lowest rates seen amongst patients nearest to death, suggesting that the 'worst case scenario' feared by many who wish to see $\mathrm{AD}$ legalised may not come to pass, and many find quality of life in circumstances which might not seem likely to those who are well. Links between attitudes and some symptoms and the possibility of changing views in response to symptom severity are discussed, with the suggestion that many would see $\mathrm{AD}$ as a reassuring future possibility rather than an option for their current situation. Participants seemed to welcome the opportunity to discuss this topic.

\section{MISSED OPPORTUNITIES TO DIE AT HOME: AN ANALYSIS OF LIVER DISEASE DEATHS IN THE VOICES SURVEY}

Roberta I Jordan, Yousuf ElMokhallalati, Lynsey Corless, Michael I Bennett. University of Leeds, University of Hull

\subsection{6/spcare-2020-PCC.88}

Background Liver disease was the 2nd most common cause of death in working age adults in 2018 with deaths increasing four-fold in the last 4 decades. Patients with advanced disease are often unaware of their prognosis and report significant unaddressed symptoms. Over $70 \%$ of deaths occur in hospital but it is unclear how avoidable admissions are and whether this reflects patients' preferences. We aimed to compare bereaved carers' perceptions of the quality of end of life care for patients with non-malignant liver disease (NMLD), malignant liver disease (MLD) and other non-malignant diseases (ONMD).

Methods We conducted an analysis of individual-level data from the VOICES National Bereavement Survey. This dataset included 110,311 completed surveys from bereaved carers, related to a stratified random sample of 246,763 deaths registered in England 2011-2015. We compared demographics, access to specialist palliative care (SPC), place of death and overall quality of care.

Results NMLD and MLD patients had greater levels of deprivation than ONMD patients. More patients with NMLD received home and hospice SPC compared with ONMD, but less than for MLD ( $11 \%$ vs. $7 \%$ vs. $55 \%$ respectively). A higher proportion of NMLD patients die in hospital than ONMD and MLD patients $(74 \%$ vs. $53 \%$ vs. $38 \%$ respectively), despite their carers reporting $89 \%$ had expressed a preference to die at home. Comparing with ONMD and MLD, carers of NMLD patients were less likely to rate the overall quality of care in the last 3 months of life as outstanding or excellent.

Conclusions It is concerning that carers report the quality of end of life care for patients with non-malignant liver disease to be worse than with other diseases. Further research should focus on achieving integration of hepatology and community services, including SPC, to prevent unwanted hospital admissions and better facilitate care and death at home.

\section{OPIATES AND SEDATIVES PRESCRIPTION IN CCC - A COMPARISON WITH BEST PRACTICE AND THE GOSPORT MEMORIAL ENQUIRY}

Despoina-Elvira Karakitsiou, Daniel Monnery. Clatterbridge Cancer Center

\subsection{6/spcare-2020-PCC.89}

Background The Gosport Independent Panel was set up to address concerns raised by families about the initial care of their relatives in Gosport War Memorial Hospital, where discrepancies were discovered over prescription of opiates and syringe drivers. This audit was created to review opiate/sedative medications prescription in EOL patients in CCC 